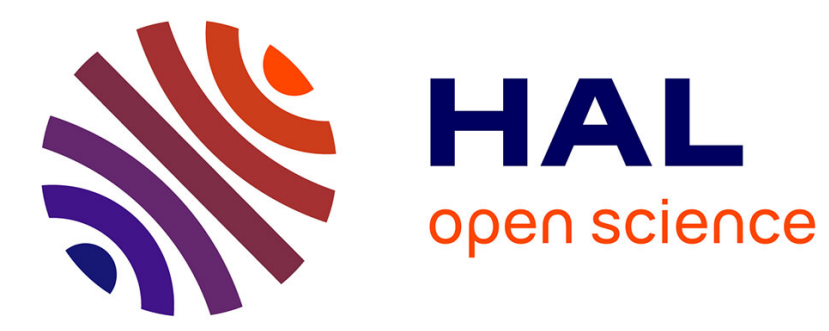

\title{
Probabilistic model definition for physiological state monitoring
}

\author{
Laure Amate, Florence Forbes, Julie Fontecave-Jallon, Benoît Vettier, \\ Catherine Garbay
}

\section{> To cite this version:}

Laure Amate, Florence Forbes, Julie Fontecave-Jallon, Benoît Vettier, Catherine Garbay. Probabilistic model definition for physiological state monitoring. SSP 2011 - Statistical Signal Processing Workshop, Jun 2011, Nice, France. pp.457-460, 10.1109/SSP.2011.5967730 hal-00742161

\section{HAL Id: hal-00742161 \\ https://hal.science/hal-00742161}

Submitted on 16 Oct 2012

HAL is a multi-disciplinary open access archive for the deposit and dissemination of scientific research documents, whether they are published or not. The documents may come from teaching and research institutions in France or abroad, or from public or private research centers.
L'archive ouverte pluridisciplinaire $\mathbf{H A L}$, est destinée au dépôt et à la diffusion de documents scientifiques de niveau recherche, publiés ou non, émanant des établissements d'enseignement et de recherche français ou étrangers, des laboratoires publics ou privés. 


\title{
PROBABILISTIC MODEL DEFINITION FOR PHYSIOLOGICAL STATE MONITORING
}

\author{
Laure Amate $^{\star}$, Florence Forbes ${ }^{\dagger}$, Julie Fontecave-Jallon ${ }^{\ddagger}$, Benoît Vettier ${ }^{\star}$, Catherine Garbay ${ }^{\star}$ \\ * LIG UMR 5217/AMA team, 38041 Grenoble, France \\ † INRIA Grenoble Rhônes-Alpes - Montbonnot, 38334 Saint Ismier, France \\ $\ddagger$ TIMC-IMAG UMR 5525 / PRETA team, 38041 Grenoble, France
}

\begin{abstract}
Assessing the global situation of a person from physiological data is a well-known difficult problem. In previous work, we propose a system that does not produce a diagnosis but instead follows a set of hypotheses and decides of an alarming situation with this information. In this paper we focus on data processing part of the system taking into account the complexity and the ambiguity of the data. We propose a statistical approach with a global model based on Hidden Markov Model and we present data models that rely on classical physiological parameters and expert's knowledge. We then learn a model that depends on the person and its environment, and we define and compute confidence values to assess the plausibility of hypotheses.
\end{abstract}

Index Terms - Graphical model, HMM, Physiological data, Context representation.

\section{INTRODUCTION}

The work presented in this paper is a contribution to the problem of identifying a person situation based on the monitoring of activity and physiological sensors. Previous works $[1,2]$ clearly establish that it is a difficult task due to the ambiguity of the data and the impossibility to directly interpret them. To solve this problem, they propose to take into account some contextual knowledge based on the idea that a person physiology is usually influenced by the environmental conditions and its activities. In [3], we propose a multi-agent system that takes into account the context and draw a set of plausible hypotheses about the person's situation. The specification of such a situation, also referred in [3] as micro-scenario, requires the definition of a person state. A person state is determined via a couple of variables: an activity state denoted by $E_{\alpha}$ and a physiological state denoted by $E_{\varphi}$. In [3], a situation is then defined as a given set of values for this couple. For instance a typical situation could correspond to a "Coffee break" which would be characterized by different possible values of $\left(E_{\alpha}, E_{\varphi}\right)$ such as $E_{\alpha}=$ "seating" and $E_{\varphi}=$ "digestion" if the coffee break is just after lunch or $E_{\alpha}=$ "walking" and $E_{\varphi}=$ "basal" it the person is in its basal state but walking while drinking. Physiology or activity states correspond to local views of the situation, they are easier to model and to link to the observed data but they are not directly the quantities of interest which are the situations at a more global level.

The system operates by navigating between plausible hypotheses for states and for micro-scenarios. To do so it requires to evaluate their "plausibility". We thus assign to each hypothesis a confidence value based on observed data. In this paper we focus on the data processing part of the system that aims at (1) learning the unknown model as an off-line process, and then (2) computing the confidence values, on-line.

In $[2,1]$, authors relies on simple data model such as interval or linear models to infer the person's situations. More recently [4] proposes to extract some features from physiological data and to classify them using a risk criterion. $\mathrm{Nu}$ merous studies propose very complex signal processing techniques for physiological data modeling such as heart rate values $[5,6]$ without considering context-dependencies. In this paper we propose a model for the data that is adaptive to the subject and takes into account the environmental conditions.

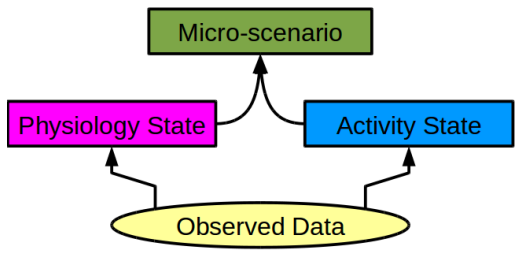

Fig. 1. Different abstraction levels.

\section{OBSERVED AND MISSING VARIABLES}

In this section, we propose a model for dependencies between unknown states and observed data based on Hidden Markov Model (HMM) [7], widely used in speech recognition.

Observed variables. At each time $t$, we observe data, $Y(t)$, that are measurements of two different types of sensors: the activity data noted $Y_{\alpha}(t)$, and the physiological data noted $Y_{\varphi}(t)$, such that $Y(t)=\left(Y_{\alpha}(t), Y_{\varphi}(t)\right) . \quad Y_{\alpha}(t)$ is a tridimensional variable provided by a tri-axis accelerometer and $Y_{\varphi}(t)$ is a couple of one-dimensional variables: the heart rate and the breath frequency noted respectively $F_{c}(t)$ and $F_{b}(t)$ : $Y_{\varphi}(t)=\left(F_{c}(t), F_{b}(t)\right)$. We denote $Y(1: T)^{1}$ the whole sequence of observations from time $t=1$ to $t=T$.

\footnotetext{
${ }^{1} u(1: t)=(u(1) u(2) \cdots u(t))$.
} 
Context variables. We introduce contextual information in our model in the form of two variables: $\mathcal{C}=(\mathcal{P}, \mathcal{E})$ where $\mathcal{P}$ denotes the subject and $\mathcal{E}$, the environmental conditions. $Y_{\alpha}(t), t \in\{1 \cdots T\}$, are physical variables and are assumed to be independent of $\mathcal{C}$ that act only on the physiology of the person. For instance, altitude affects breathing and age affects cardiac function.

Missing variables. The system relies on two levels: microscenario and state, and we divide the state level into two different types: one for activity and one for physiology (see Figure 1). They are unobserved variables and we denote them $E_{\alpha}(t)$ and $E_{\varphi}(t)$ respectively for the activity state and physiology state at time $t$. They reflect the true activity and physiology states of a subject.

We choose a discrete description of $E_{\alpha}(t)$ : for all $t \in$ $\{1 \cdots T\}, E_{\alpha}(t) \in\left\{1,2, \cdots, M_{\alpha}\right\}$. For example, $E_{\alpha}(t)=1$ corresponds to the "inactive" state while $E_{\alpha}(t)=M_{\alpha}$ corresponds to the activity state "Intense movement".

The physiological state is more complex because we consider that multiple physiological elementary state may occur simultaneously, a typical example being the "digestion" that may occur together with "basal" or "sleep". We thus consider $E_{\varphi}(t)$ to be a set of elementary states noted $e_{i}(t), i \in$ $\left\{1 \cdots M_{\varphi}\right\}$ and $E_{\varphi}(t)$ is a binary vector of size $M_{\varphi}: E_{\varphi}(t)=$ $\left[e_{1}(t) e_{2}(t) \cdots e_{M_{\varphi}}(t)\right]$, where $e_{i}(t) \in\{0 ; 1\}$ (0 corresponding to inactive state and 1 to active state). For instance, with three elementary states "basal", "sleep", "digestion", $E_{\varphi}(t)=$ [100] is a "basal" state and $E_{\varphi}(t)=[011]$ is a "sleep and digest" state. Theoretically $E_{\varphi}(t)$ can take $2^{M_{\varphi}}$ values. In fact some configurations are impossible because many elementary states are incompatible: for example, "exercise" and "sleep" can not be simultaneously active. We represent these incompatibilities with a graph as shown in figure 2 . The physiological state space is reduced to $N_{\varphi}$ states where $N_{\varphi}$ is establish with combinatorial rules. For example in figure $2, N_{\varphi}=10$.

We note $E_{\alpha}(1: T)$ and $E_{\varphi}(1: T)$ respectively the activity and the physiology states from time $t=1$ to $t=T$.

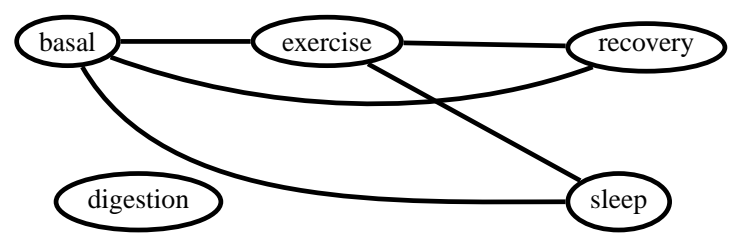

Fig. 2. Graph representation of incompatibilities between elementary physiological states.

We thus propose a graphical model with two chains, one for each unobserved state, and two different dependencies for the data : activity data depend only on activity state while physiological data depend on both activity and physiology state (see figure 3). This is a variant of Factorial HMM [8] where data are split into two distinct sets.

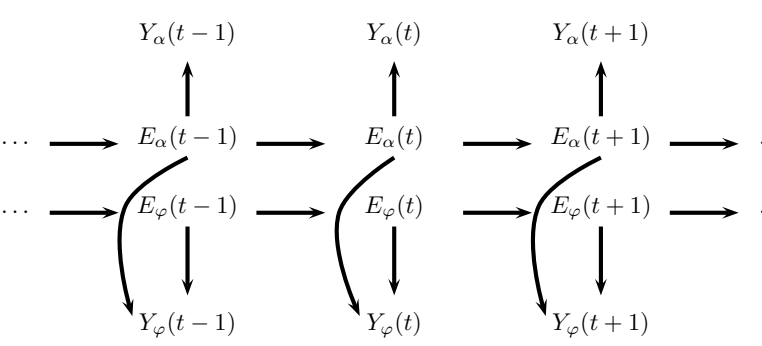

Fig. 3. Physiological and activity data graphical model.

\section{A CONTEXT CONDITIONAL JOINT MODEL}

The joint model of our observed and missing variables is defined conditionally to the context variables $\mathcal{C}$ :

$$
\begin{aligned}
& p\left(Y_{\alpha}(1: T), Y_{\varphi}(1: T), E_{\alpha}(1: T), E_{\varphi}(1: T) \mid \mathcal{C}\right)= \\
& \quad p\left(Y_{\alpha}(1: T), Y_{\varphi}(1: T) \mid E_{\alpha}(1: T), E_{\varphi}(1: T), \mathcal{C}\right) p\left(E_{\alpha}(1: T), E_{\varphi}(1: T) \mid \mathcal{C}\right) .
\end{aligned}
$$

Missing data model. The model takes into account the temporal dependencies and both states are time-dependent. More specifically we define $p\left(E_{\alpha}(1: T), E_{\varphi}(1: T) \mid \mathcal{C}\right)$ as an homogeneous Markov chain whose state space is discrete and with the following transition matrix. We assume that activity and physiology states transitions are independent such that:

$$
\begin{gathered}
P\left(\left(E_{\alpha}(t), E_{\varphi}(t)\right)=(i, u) \mid\left(E_{\alpha}(t-1), E_{\varphi}(t-1)\right)=(j, v) ; \mathcal{C}\right)= \\
P\left(E_{\alpha}(t)=i \mid E_{\alpha}(t-1)=j ; \mathcal{C}\right) P\left(E_{\varphi}(t)=u \mid E_{\varphi}(t-1)=v ; \mathcal{C}\right)= \\
T_{\alpha}\left(E_{\alpha}(t-1)=i, E_{\alpha}(t)=j\right) \times T_{\varphi}\left(E_{\varphi}(t-1)=u, E_{\varphi}(t)=v\right),
\end{gathered}
$$

where $T_{\alpha}$ and $T_{\varphi}$ denote the transition matrices. The transition matrices can be part of the unknown parameter of the model that must be estimated on data sets. However, they introduce a large number of unknown parameters in the model and we want to add some expert's knowledge about these transitions. For example, "recovery" will always follow "exercise". So we prefer to treat them as fixed parameters in this preliminary work, knowing that we can relax this assumption and learn them from the data.

Observed data model. The physiological and activity data are assumed to be conditionally independent:

$$
\begin{aligned}
& p\left(Y_{\alpha}(1: T), Y_{\varphi}(1: T) \mid E_{\alpha}(1: T), E_{\varphi}(1: T), \mathcal{C}\right)= \\
& \quad p\left(Y_{\alpha}(1: T) \mid E_{\alpha}(1: T), E_{\varphi}(1: T), \mathcal{C}\right) p\left(Y_{\varphi}(1: T) \mid E_{\alpha}(1: T), E_{\varphi}(1: T), \mathcal{C}\right) .
\end{aligned}
$$

For each $t$, we model $Y_{\alpha}(t)$ as a trivariate Gaussian variable whose mean and covariance matrix depend on the activity state $E_{\alpha}(t)$ :

$$
\left(Y_{\alpha}(t) \mid E_{\alpha}(t)=k ; \mathcal{C}\right) \sim \mathcal{N}_{3}\left(\mu_{\alpha}(k) ; \Sigma_{\alpha}(k)\right)
$$

The corresponding unknown parameters to be estimated are denoted by $\theta_{\alpha}$ with:

$$
\theta_{\alpha}=\left(\left\{\mu_{\alpha}(k), \Sigma_{\alpha}(k)\right\}, k=1 \cdots M_{\alpha}\right) .
$$

By contrast, the physiological data must take into account the context $\mathcal{C}$ and, in addition, we assume that they do not actually depend directly on $E_{\alpha}$ and $E_{\varphi}$ but via some discrete 
quantity $h\left(E_{\alpha}, E_{\varphi}\right)$ where $h$ is a function taking a finite number of values in $\mathcal{H}=\{0 \ldots H\}$. The possible values for $h$ can be interpreted as different intensity levels. Typically, different combinations of $E_{\alpha}$ and $E_{\varphi}$ can lead to the same level $h \in \mathcal{H}$. For instance, "exercise" at a low level of activity and "recovery and digestion" at medium level of activity yield similar physiological data. The function $h$ is assumed to be defined a priori by experts. It follows that,

$$
\left(Y_{\varphi}(t) \mid E_{\alpha}(t)=k, E_{\varphi}(t)=u ; \mathcal{C}\right) \sim \mathcal{N}_{2}\left(\mu_{\varphi}(\mathcal{C}, h(k, u)), \Sigma_{\varphi}(\mathcal{C}, h(k, u))\right) .
$$

The dependence on the context variables can then be further specified. The heart rate and breath frequency are made of one part which is only person specific to which is added a quantity that depends both on the context variables and person state. More specifically, we consider two well-known physiological quantities the maximal heart rate $F_{c}^{\max }$ and the basal breath frequency $F_{b}^{b a s a l}$ to which is added an adjustment function detailed in section 5. The maximal heart rate $F_{c}^{\max }$ and the basal breath frequency $F_{b}^{b a s a l}$ are person specific but do not depend on the other context variables nor on the person state.

$$
\mu_{\varphi}(\mathcal{C}, h)=\left[\begin{array}{l}
m_{c}(\mathcal{C}, h) \\
m_{b}(\mathcal{C}, h)
\end{array}\right]=\left[\begin{array}{l}
F_{c}^{\max }(\mathcal{P})-f_{c}(\mathcal{C}, h) \\
F_{b}^{\text {basal }}(\mathcal{P})+f_{b}(\mathcal{C}, h)
\end{array}\right] .
$$

Similarly we fix a model for the covariance matrix assuming zero covariance terms:

$$
\Sigma_{\varphi}(\mathcal{C}, h)=\left[\begin{array}{cc}
\sigma_{c}(\mathcal{C}, h) & 0 \\
0 & \sigma_{b}(\mathcal{C}, h)
\end{array}\right]
$$

Finally, for context variable $\mathcal{C}$, the unknown physiological parameters are:

$\theta_{\varphi}(\mathcal{C})=\left(F_{c}^{\text {max }}(\mathcal{P}), F_{b}^{\text {basal }}(\mathcal{P}), f_{c}(\mathcal{C}, h), f_{b}(\mathcal{C}, h), \sigma_{c}(\mathcal{C}, h), \sigma_{b}(\mathcal{C}, h) ;\right.$ for all $\left.h\right)$

The whole set of parameters for the model $\theta$ is:

$$
\theta=\left(\theta_{\alpha}, \theta_{\varphi}(\mathcal{C})\right) \text {. }
$$

\section{PARAMETERS IDENTIFICATION AND CONFIDENCE COMPUTATION}

We face two tasks: estimate the parameters to identify the model, and define and compute states confidence values.

Parameter identification is an off-line process and within this graphical framework, we choose a Maximum Likelihood (ML) criterion such that:

$$
\hat{\theta}=\arg \max _{\theta} p(Y(1: T) \mid \theta) .
$$

For problems with hidden states, because the likelihood $p(Y(1: T) \mid \theta)$ is untractable, we use a numerical approach based on Expectation-Maximization (EM) algorithm [9] to infer the estimate $\hat{\theta}$. The computation is performed using the Bayes Net toolbox for Matlab [10].

We now define for each time $t$ the confidence value for the states as their posterior probabilities:

$$
\begin{aligned}
& c\left(E_{\alpha}(t)=k\right) \triangleq p\left(E_{\alpha}(t)=k \mid Y(1: t), \theta\right) \\
& \quad=\sum_{u} p\left(E_{\alpha}(t)=k, E_{\varphi}(t)=u \mid Y(1: t), \theta\right),
\end{aligned}
$$

$$
\begin{aligned}
& c\left(e_{j}(t)=1\right) \triangleq p\left(e_{j}(t)=1 \mid Y(1: t), \theta\right) \\
& \quad=\sum_{k} \sum_{u \mid u(j)=1} p\left(E_{\alpha}(t)=k, E_{\varphi}(t)=u \mid Y(1: t), \theta\right)
\end{aligned}
$$

with $k \in\left\{1 \cdots M_{\alpha}\right\}$ and $u$ is a binary vector of size $M_{\varphi}$ with element $j$ noted $u(j)$.

\section{PRELIMINARY RESULTS}

We illustrate our model with the following simulated data.

We first describe the adjustment functions with $H=5$ :

$$
f_{c}(\mathcal{C}, h)=\left\{\begin{array}{cl}
10 & \text { if } h=5 \\
20 & \text { if } h=4 \\
\cdots & \cdots \\
50 & \text { if } h=1 \\
\gamma(\mathcal{P}) & \text { if } h=0
\end{array} \quad, f_{b}(\mathcal{C}, h)=\left\{\begin{array}{cl}
0 & \text { if } h=0 \\
\Delta & \text { if } h=1 \\
2 \Delta & \text { if } h=2 \\
\cdots & \cdots \\
5 \Delta & \text { if } h=5
\end{array},\right.\right.
$$

where $\gamma(\mathcal{P})=F_{c}^{\max }(\mathcal{P})-F_{c}^{\text {rest }}(\mathcal{P})$ and $\Delta=\frac{60-F_{b}^{\text {basal }}(\mathcal{P})}{5}$.

We note that estimating $F_{c}^{\max }$ requires very specific experimental settings not available for us and we use the wellknown deterministic functions:

$$
F_{c}^{\max }(P)=\left\{\begin{array}{cl}
220-\operatorname{age}(\mathcal{P}) & \text { for men } \\
206-88 \% \operatorname{age}(\mathcal{P}) & \text { for women }
\end{array}\right.
$$

We simulate a sequence of data for a men aged 32 with $F_{c}^{\text {rest }}=80 \mathrm{bpm}$ and $F_{b}^{\text {basal }}=14$ breaths $/$ min: $E_{\alpha}(1$ : $200)=$ "inactive", $E_{\varphi}(1: 200)=$ "basal" ; $E_{\alpha}(201$ : $500)=$ "medium movement", $E_{\varphi}(201: 500)=$ "exercise" ; $E_{\alpha}(501: 600)=$ "inactive", $E_{\varphi}(501: 600)=$ "recovery" ; $E_{\alpha}(601: 800)=$ "inactive", $E_{\varphi}(601: 800)=$ "basal". Values of corresponding $h(\cdot, \cdot)$ are given in the second column in Table 1.

The goal is to (i) learn the parameter with ML criterion and correctly recover the model ; (ii) compute confidence values for all states.

We display the estimated parameters obtained in Table 1. We are able to recover the parameters except for the situation where $E_{\alpha}=$ "inactive" and $E_{\varphi}=$ "recovery", because data could correspond to another situation: $E_{\alpha}=$ "medium movement", $E_{\varphi}=$ "basal". This is due to the fact that $h(\cdot, \cdot)$ is a surjection. In future work we must include this ambiguity in the estimation criterion.

In the following, we use the model described above with $M_{\alpha}=4$ and the set of physiology states of figure 2. With the same simulated data and with parameters correctly estimated, we compute the confidence values of states (see figures 4 and 5). Figure 4 shows confidence values of activity states exhibiting that between $t=200$ and $t=500$ there is no clear choice between "medium movement" and "intense movement", explaining that we want to maintain both hypotheses in our monitoring system. Similarly, for physiology states, figure 5 shows that if we want to select the real state then maximizing the confidence values yields a wrong result. 


\begin{tabular}{|c|c|c|c|c|c|c|c|c|c|c|c|c|c|}
\hline \multirow{2}{*}{ situations } & & \multicolumn{12}{|c|}{ Model parameters } \\
\hline & $\mathrm{h}$ & $m_{c}$ & $\hat{m}_{c}$ & $m_{b}$ & $\hat{m}_{b}$ & $\mu_{\alpha}$ & $\hat{\mu}_{\alpha}$ & $\sigma_{c}$ & $\hat{\sigma_{c}}$ & $\sigma_{b}$ & $\hat{\sigma}_{b}$ & $\Sigma_{\alpha}$ & $\hat{\Sigma}_{\alpha}$ \\
\hline $\begin{array}{c}\text { basal } \\
\text { inactive }\end{array}$ & 0 & 80 & 79.96 & 14 & 13.98 & {$\left[\begin{array}{l}560 \\
440 \\
580\end{array}\right]$} & {$\left[\begin{array}{l}560.05 \\
440.05 \\
580.09\end{array}\right]$} & 4 & 3.80 & 2 & 1.97 & {$\left[\begin{array}{lll}5 & 0 & 0 \\
0 & 5 & 0 \\
0 & 0 & 5\end{array}\right]$} & {$\left[\begin{array}{ccc}5.08 & 0 & 0 \\
0 & 5.44 & 0 \\
0 & 0 & 4.93\end{array}\right]$} \\
\hline $\begin{array}{l}\text { exercise } \\
\text { medium } \\
\text { movement }\end{array}$ & 4 & 168 & 168.52 & 50.8 & 50.86 & {$\left[\begin{array}{l}560 \\
440 \\
580\end{array}\right]$} & {$\left[\begin{array}{l}559.99 \\
440.20 \\
579.86\end{array}\right]$} & 15 & 14.63 & 8 & 8.61 & {$\left[\begin{array}{ccc}30 & 0 & 0 \\
0 & 30 & 0 \\
0 & 0 & 30\end{array}\right]$} & {$\left[\begin{array}{ccc}23.53 & 0 & 0 \\
0 & 22.71 & 0 \\
0 & 0 & 23.64\end{array}\right]$} \\
\hline $\begin{array}{l}\text { recovery } \\
\text { inactive }\end{array}$ & 2 & 148 & 163.52 & 32.4 & 44.98 & {$\left[\begin{array}{l}560 \\
440 \\
580\end{array}\right]$} & {$\left[\begin{array}{l}560.05 \\
440.05 \\
580.09\end{array}\right]$} & 8 & 0.93 & 4 & 1.08 & {$\left[\begin{array}{lll}5 & 0 & 0 \\
0 & 5 & 0 \\
0 & 0 & 5\end{array}\right]$} & {$\left[\begin{array}{ccc}5.08 & 0 & 0 \\
0 & 5.44 & 0 \\
0 & 0 & 4.93\end{array}\right]$} \\
\hline
\end{tabular}

Table 1. Model and learned parameters for the simulated sequence.

We thus prefer to select a set of hypothesis to monitor the subject situation: for example, for $t \in[200,500]$, we maintain "recovery", "sleep" and "exercise" states.

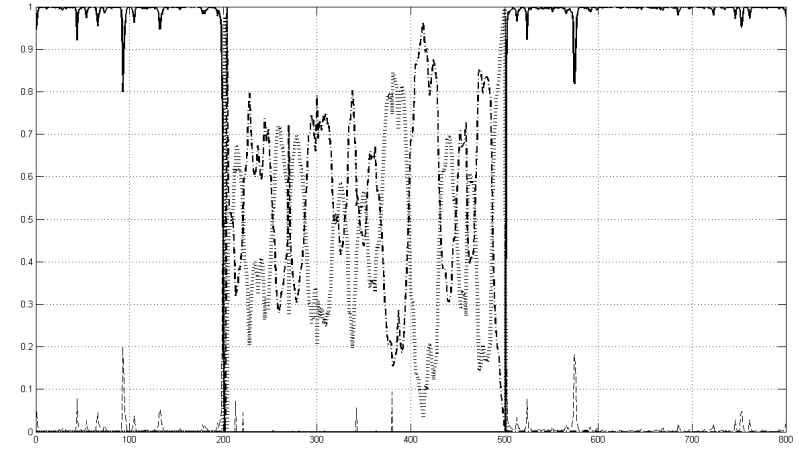

Fig. 4. Confidence values of activity states, $t \in[1 \cdots T]$ : "inactive" (line), "low movement" (dash), "medium movement" (dot), "intense movement" (dash and dot).

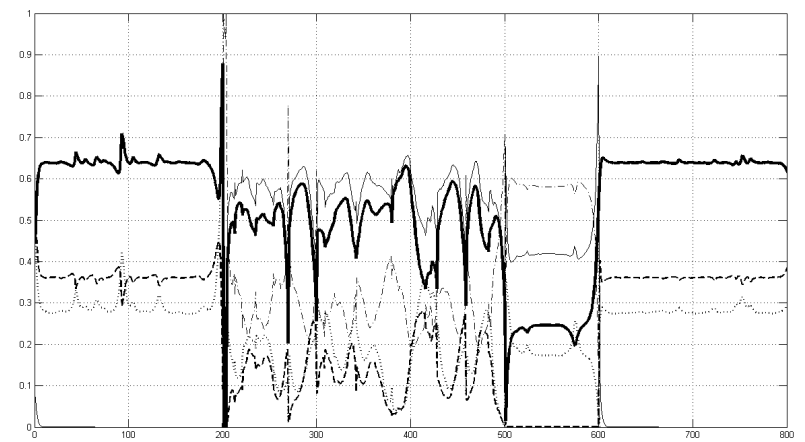

Fig. 5. Confidence values of physiology states, $t \in[1 \cdots T]$ : "basal" (dash), "exercise" (dash and dot), "recovery" (thin plain), "sleep" (large plain), "digestion" (dot).

\section{CONCLUSION}

We presented a dynamic Bayesian network based on HMM to model the physiological state of a subject allowing thus to select a set of "plausible" hypotheses of the real situation. Our model takes into account the specificity of the personal physiology and the environmental conditions. This allows us to learn parameters as an off-line process and then to compute posterior probability for each state. The overall goal is to use confidence values in the global multi-agent system that manages the set of hypotheses. We present here some preliminary results. Future work includes the adaption of the learning algorithm, the validation on real data and the use of approximation techniques to handle more complex models.

Thanks. PRETA/TIMC experts participated in elaborating physiological models. This work is funded by DGA project "SuPerCo".

\section{REFERENCES}

[1] U. Anliker et al. "Amon: a wearable multiparameter medical monitoring and alert system," IEEE T. Inf. Technol. Biomedicine, vol. 8, no. 4, p. 415-427, 2004.

[2] A.-S. Silvent, M. Dojat, and C. Garbay, "Multi-level temporal abstraction for medical scenario construction," Int. J. Adapt. Control., vol. 19, p. 377-394, 2005.

[3] B. Vettier, L. Amate, C. Garbay, and P. Baconnier, "Une architecture multi-agents adaptative pour le suivi personnalisé de la physiologie du combattant," in JFSMA ,2010.

[4] D. Apiletti, E. Baralis, G. Bruno, and T. Cerquitelli, "Real-time analysis of physiological data to support medical applications," Trans. Info. Tech. Biomed., vol. 13, pp. 313-321, May 2009.

[5] J.M. Bardet, V. Billat, and I. Kammoun, "Modélisation des fréquences cardiaques instantanées durant un marathon et estimation de leurs paramètres fractals," J. Soc. Fr. Stat. \& Rev. Stat. Appl., vol. 150, pp. 101-126, 2009.

[6] O. Stegle, S. V. Fallert, D. J. MacKay, and S. Brage, "Gaussian process robust regression for noisy heart rate data.," IEEE T. Bio-Med. Eng., vol. 55, no. 9, pp. 2143-2151, September 2008.

[7] L. Rabiner and B. Juang, "An introduction to hidden markov models," ASSP Magazine, IEEE, vol. 3, no. 1, pp. 4-16, January 1986.

[8] Z. Ghahramani and M.I. Jordan, "Factorial hidden markov models," Machine Learning, vol. 29, pp. 245-273, 1997.

[9] O. Cappé, E. Moulines, and T. Ryden, Inference in Hidden Markov Models, Springer-Verlag, 2005.

[10] Kevin P. Murphy, "The bayes net toolbox for matlab," Computing Science and Statistics, vol. 33, pp. 2001, 2001. 